\title{
Erratum
}

Cold Spring Harb Protoc 2017; doi: 10.1101/pdb.prot098558

\section{Erratum: Detergent Lysis of Tissue Culture Cells for Immunoprecipitation}

James DeCaprio and Thomas O. Kohl

When the above protocol was published, multiple reagents in the Nonidet P-40 Lysis Buffer and RIPA Lysis Buffer recipes and/or their volumes and concentrations were incorrect. The publisher apologizes for any confusion related to these errors, which appear to have been inadvertently introduced when the protocol was formatted for publication. The PDF version of the protocol and the HTML versions of the Nonidet P-40 Lysis Buffer (doi: 10.1101/pdb.rec101410) and RIPA Lysis Buffer (10.1101/pdb .rec101428) recipes have been replaced with the corrected recipes shown below. The originally published versions of the recipes are also included.

\section{CORRECTED RECIPES}

Nonidet P-40 Lysis Buffer

Reagent

Final concentration

$\mathrm{NaCl}$

$150 \mathrm{~mm}$

Nonidet P-40

$1 \%$

Tris ( $\mathrm{pH}$ 8.0)

$50 \mathrm{~mm}$

Prepared Nonidet P-40 lysis buffer should be aliquoted and stored at $-20^{\circ} \mathrm{C}$. Add protease and/or phosphatase inhibitors to a thawed aliquot before immediate use. Discard and do not freeze again.

RIPA Lysis Buffer

Reagent

Final concentration

$\mathrm{NaCl}$

Nonidet P-40

$150 \mathrm{~mm}$

DOC

$1 \%$

SDS

$0.5 \%$

Tris ( $\mathrm{pH} 7.4)$

$0.1 \%$

Prepared RIPA buffer should be aliquoted and stored at $-20^{\circ} \mathrm{C}$. Add protease and/or phosphatase inhibitors to a thawed aliquot before immediate use. Discard and do not freeze again. One milliliter of buffer is sufficient to lyse approximately 5 million cells.

(C) 2021 Cold Spring Harbor Laboratory Press

Cite this erratum as Cold Spring Harb Protoc; doi:10.1101/pdb.err107714 
Nonidet P-40 Lysis Buffer

Reagent

Volume per $10 \mathrm{~mL}$ of solution (v/v) Final concentration

$\mathrm{NaCl}(150 \mathrm{~mm})$

Nonidet P-40 (1\%)

Tris (50 mM, pH 7.4)

$\mathrm{MgCl}_{2}(10 \mathrm{~mm})$

$\mathrm{ddH}_{2} \mathrm{O}$

$\begin{array}{rr}0.66 \mathrm{~mL} & 10 \mathrm{~mm} \\ 0.1 \mathrm{~mL} & 1 \% \\ 2 \mathrm{~mL} & 10 \mathrm{~mm} \\ 3 \mathrm{~mL} & 3 \mathrm{~mm}\end{array}$

Prepared Nonidet P-40 lysis buffer should be aliquoted and stored at $-20^{\circ} \mathrm{C}$. Add protease and/or phosphatase inhibitors to a thawed aliquot before immediate use. Discard and do not freeze again.

RIPA Lysis Buffer

Reagent Volume per $10 \mathrm{~mL}$ of solution (v/v) Final concentration

$\mathrm{NaCl}(1 \mathrm{M})$

Nonidet P-40 (1\%)

Sodium deoxycholate (DOC)

$(0.5 \%)$

SDS $(0.1 \%)$

Tris (50 mm, pH 7.4)

$\mathrm{ddH}_{2} \mathrm{O}$

$1.5 \mathrm{~mL}$

$0.1 \mathrm{~mL}$

$150 \mathrm{~mm}$

$0.05 \mathrm{~mL}$

$1 \%$

$0.01 \mathrm{~mL}$

$5 \mathrm{~mL}$

Add to $10 \mathrm{~mL}$

Prepared RIPA buffer should be aliquoted and stored at $-20^{\circ} \mathrm{C}$. Add protease and/or phosphatase inhibitors to a thawed aliquot before immediate use. Discard and do not freeze again. This amount is sufficient for approximately 5 million cells per $1 \mathrm{~mL}$ of reagent. 


\section{Erratum: Detergent Lysis of Tissue Culture Cells for Immunoprecipitation}

James DeCaprio and Thomas O. Kohl

Cold Spring Harb Protoc; doi: 10.1101/pdb.err107714

\begin{tabular}{cc}
\hline $\begin{array}{r}\text { Email Alerting } \\
\text { Service }\end{array}$ & Receive free email alerts when new articles cite this article - click here. \\
\hline $\begin{array}{c}\text { Subject } \\
\text { Categories }\end{array}$ & Browse articles on similar topics from Cold Spring Harbor Protocols. \\
\hline
\end{tabular}

